\title{
Reflections from the Ground: I Know What I'm doing but am I Doing the Right Thing?
}

\section{Wolman $\mathbf{M}^{*}$}

Program Manager, New Jersey Medical School, USA

*Corresponding author: Mark Wolman, Program Manager, Global Tuberculosis Institute, Rutgers, The State University, USA, Tel: 973-508-5919; Email: wolmanms@njms.rutgers.edu

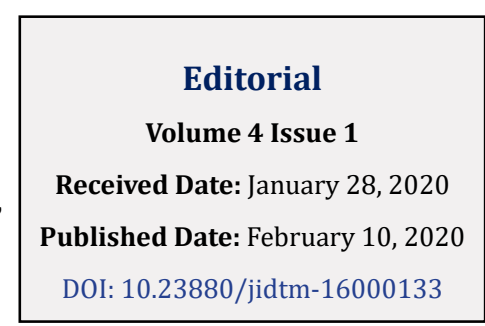

Abbreviations: CDC: Centers for Disease Control and Prevention; TB: Tuberculosis; AFB: Acid-Fast Bacilli.

\section{Editorial}

William Osler, a Canadian-born physician from the late 1800 s and early 1900s was quoted as saying "medicine is a science of uncertainty and an art of probability". However, by inserting my own words into this statement it would now read "a contact investigation, most notably a congregate setting investigation, is a science of uncertainty and an art of probability".

In my opinion, I believe there is an unspoken yet undeniable embrace of uncertainty and probability guiding us through congregate setting investigations as we attempt, to the best of our ability, to identify those contacts requiring medical evaluations. As we know, in the United States the Centers of Disease Control and Prevention (CDC) offers important yet basic parameters in the form of guidance but does not provide technical instructions found in national guidelines, protocols, standards of practice, or recommendations in the step-by-step process of these large-scale investigations. And as there is now increasing international interest in contact investigations beyond the household uncertainty and probability undoubtedly will likewise be prevalent. In the United States CDC entrusts state and local health departments to develop their own standards of how to proceed during these investigations. While this does free us from being tethered to national standards, it does however, potentially create a national atmosphere of variability from state to state and, at times, even within states in how TB programs formulate decisions in differentiating and identifying high risk from low risk contacts in addition to the worried well. Yet, I do understand why there are no national standards because one size does not fit all.

Every TB patient, every environment of exposure, every duration of exposure and every TB contact should be regarded as unique requiring its own distinct set of analysis. So, as a result of national variability combined with ambiguous and inconclusive evidence regarding TB transmission the decisions we make in the field during these investigations essentially are built on a foundation of uncertainty and of probability. As an example, it is noteworthy to mention the apparent variability in the application of the number of exposure hours that help distinguish a high risk (high priority) contact from a low risk (low priority) contact and from the worried well. For instance, in one document (MMWR 2005) the CDC offers for consideration one set of criteria at 120 exposure hours per month during a typical infectious period for the pulmonary patient without cavitary disease (excluding bacteriology and cough history). This indicates an average 30 exposure hours per week and depending upon the number of exposure days in a particular week there may be a potential wide range of exposure hours per day. Other expert opinions (Journal of Infectious Diseases) place the exposure hours at $>180$ during the infectious period with $>15$ hours per week. Again, depending on the number of exposure days in a particular week, there may be a wide range of exposure hours per day. Additionally, I have been told anecdotally other programs may use 1 hour per day or 7 hours per week as their recommended guidelines.

Finally, it is also worth mentioning based upon consultation requests we receive from our region that are collected through the medical consultation line at the NJMS Global TB Institute at Rutgers University discussion of congregate setting investigations typically center on the complex and unclear issue of contact identification based on not only hours but on environments of exposure which presents as another important element in the subsequent identification of high and low risk contacts. As such, looking beyond the frequency and duration of exposure hours allows the investigator to assess the environment of exposure 
during the infectious period. For example, a patient who is sputum AFB smear positive (4+) with cavitary disease and cough for 2 months who is employed as a full time worker with 20 co-workers in a warehouse measuring 200,000 sq. $\mathrm{ft}$. (no use of break room) may be less of a priority than a part time employee in an office measuring $600 \mathrm{sq}$. $\mathrm{ft}$. who is sputum AFB smear positive (2+) with non-cavitary disease and cough for 2 weeks. If culture confirmed both patients would be considered infectious but one environment (office) may take precedent over the other in terms of the potential for transmission at the workplace. In this example defined hours of exposure may play less of a role in identifying contacts than the actual environment of exposure.

Over the years I have developed a fair amount of experience and self-confidence in addressing and working through many types of congregate setting investigations. However, in spite of this, I am acutely aware of the uncertainties and presumptions we make that move us through these investigations. As a result, in the midst of many investigations, with all my experience and self-confidence, I find myself taking pause and wondering if the decisions I make are indeed correct. And so the question remains in the absence of scientific based evidence regarding transmission is this the best we can do? Are we destined to continually rely on presumptions, uncertainties and probabilities in our decision-making? Will we forever be wondering and hoping we make the correct decisions? Other factors linger and call for consideration. For instance, genotyping has offered insight and has demonstrated that transmission may be occurring through casual as well as prolonged exposure. Is molecular epidemiology now suggesting we look beyond hours and environments of exposure? And what of host risk factors and strain virulence in determining the identification of contacts? I'm certain there is agreement that there is much to understand about TB transmission and the identification of contacts but the question remains do we continue to accept things as they are? If not, where do we go from here?

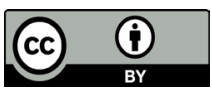

